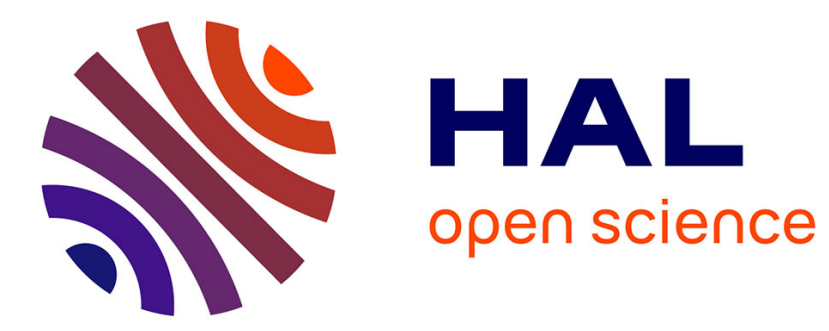

\title{
Decentralized partial-consensus control of nonholonomic vehicles overnetworks with interconnection delays
}

\author{
Mohamed Maghenem, Antonio Loria, Emmanuel Nuño, Elena Panteley
}

\section{To cite this version:}

Mohamed Maghenem, Antonio Loria, Emmanuel Nuño, Elena Panteley. Decentralized partial-consensus control of nonholonomic vehicles overnetworks with interconnection delays. ACC 2020 - American Control Conference, Jul 2020, Denver, CO, United States. 10.23919/acc45564.2020.9147569 . hal-03101503

\section{HAL Id: hal-03101503 \\ https://hal.science/hal-03101503}

Submitted on 7 Jan 2021

HAL is a multi-disciplinary open access archive for the deposit and dissemination of scientific research documents, whether they are published or not. The documents may come from teaching and research institutions in France or abroad, or from public or private research centers.
L'archive ouverte pluridisciplinaire HAL, est destinée au dépôt et à la diffusion de documents scientifiques de niveau recherche, publiés ou non, émanant des établissements d'enseignement et de recherche français ou étrangers, des laboratoires publics ou privés. 


\title{
Decentralized partial-consensus control of nonholonomic vehicles over networks with interconnection delays
}

\author{
Mohamed Maghenem Antonio Loría Emmanuel Nuño Elena Panteley
}

\begin{abstract}
We present a partial-consensus controller for a network of nonholonomic mobile robots in the presence of timevarying communication delays. The control problem that we address is that of having a group of vehicles converging to a relatively common non-specified Cartesian position value, but each having an imposed desired orientation. The vehicles are assumed to communicate over a network with a connected, undirected graph topology. The proposed decentralized control law is smooth time-varying, of the $\delta$-Persistently-Exciting class [1]. We establish uniform global asymptotic stability for the closed-loop system.
\end{abstract}

\section{INTRODUCTION}

In decentralized, or distributed, solutions to consensus problems, the control input for each node depends only on local information provided from a given set of nodes in the network called neighbors. The structure of information exchange between the neighbors is specified using a communication graph [2]. The most important challenges when proposing decentralized solutions for consensus problems rises, first, when considering a constrained communication between the neighbors. The later includes unidirectional flow of information [3], [4], unreliable communication with respect to time [5], [6], and delayed communication [7], [8], to name only few. The second important challenge is related to the individual dynamics of the nodes. That is, the node's dynamics can be linear [9], nonlinear [10], identical for all the network or heterogeneous [11], [12].

An example of strongly nonlinear network is that of nonholonomic mobile robots. Indeed, the nonholonomic restriction, in this case, prevents the applicability of smooth autonomous controllers to solve consensus problems [13]. As a consequence, different decentralized solutions for consensus problems in networks of nonholonomic mobile robots are proposed in the literature. In [14], full consensus, that is, in terms of both position and orientation is solved using discontinuous and time-invariant controllers. In [15] and [16], the consensus problem is solved using smooth timevarying control laws that lead the agents to a given formation using only their orientation. The result in [16], is extended in

M. Maghenem is with the University of California at Santa Cruz. E-mail: mmaghene@ucsc.edu. E. Nuño is with the Dept. of Computer Science CUCEI, at University of Guadalajara, Guadalajara, Mexico. Emails: emmanuel.nuno@cucei.udg.mx. A. Loría and E. Panteley are with LSS-CentraleSupelec, CNRS, 3 Rue Joliot Curie, 91192, France. E-mails: loria(panteley)@1ss.supelec.fr. E. Panteley is also with ITMO University, Kronverkskiy av. 49, Saint Petersburg, 197101, Russia.

This article was supported by the Government of Russian Federation (grant 08-08), by ECOS-Nord contract M14M02, by the ANR, under contract no. ANR-18-CE40-0010, by the Mexican CONACyT Basic Scientific Research grant, no. CB-282807, and by CEFIPRA (grant number 6001-A).
[17] in the presence of smooth time-varying communication delays while considering a complete dynamical model of the vehicles. The same problem is addressed in [18] using timevarying controllers while assuming a constant time delay affecting the flow of information between the agents. In [19] the same problem is solved in the presence of bounded disturbances with unknown dynamics in all the input channels. Most of the works proposing smooth time-varying controllers for nonholonomic vehicles, however, employ a simplified first order model that considers exclusively the kinematics and, to the best of our knowledge, none of them guarantee uniform convergence of the consensus errors, let alone uniform asymptotic stability of the consensus set.

In this paper we present a partial-consensus controller for a network of nonholonomic mobile robots and we establish uniform global asymptotic stability for the closedloop system. In particular, it is established that the positions converge towards a specified geometric pattern around an a priori unknown center, while the orientations of each vehicle converge to a specified reference. It is assumed that the vehicles communicate over a network having a connected and undirected topology graph. In addition, it is assumed that the transfer of information between agents is affected by time-varying delays which are only bounded and not necessarily differentiable. Such time delays appear naturally, e.g., when the systems communicate over wireless networks or the Internet [7].

In contrast to related literature, the vehicle's model that we consider includes both the vehicle's kinematics and dynamics. Our controller is smooth time-varying, of the $\delta$-Persistently-Exciting class - [1], [20], [21], and extends the one proposed in [22] to the case where time-varying communication delays are present. Another significant contribution of this paper is to establish uniform global asymptotic stability.

The rest of the paper is organized as follows. In the next section we describe the networked systems' model and we formulate the problem at hand. Our main result is presented in Section III and some simulation results are provided in Section IV. Concluding remarks are presented in Section V.

\section{PRoBlem FORMULATION}

Let us consider a swarm of $N$ autonomous vehicles modeled as force-controlled unicycles, that is,

$$
\begin{aligned}
\dot{x}_{i} & =v_{i} \cos \left(\theta_{i}\right) \\
\dot{y}_{i} & =v_{i} \sin \left(\theta_{i}\right) \\
\dot{\theta}_{i} & =\omega_{i}, \quad i \leq N
\end{aligned}
$$




$$
\begin{aligned}
& \dot{v}_{i}=u_{v i} \\
& \dot{\omega}_{i}=u_{\omega i},
\end{aligned}
$$

where $x_{i}$ and $y_{i}$ denote the Cartesian coordinates of a fixed point on the vehicle relative to a fixed frame, $\theta_{i}$ denotes its orientation with respect to the $X$-axis, and $v_{i}$ and $\omega_{i}$ denote the forward and angular velocities, respectively, and $\left(u_{v i}, u_{\omega i}\right)$ correspond to the control inputs. That is, it is considered that the vehicles are force-controlled. This model corresponds, in particular, to differential-drive robots, in which case, there exists a direct relation between the wheels' input torques and $\left(u_{v i}, u_{\omega i}\right)$. Other force-controlled models include dynamics equations in Lagrangian form [23], but since the latter is usually fully-actuated, it may be assumed that a preliminary feedback linearizing controller is applied to obtain (2). Indeed, for the $i$ th robot, it is assumed that $u_{v i}$ and $u_{\omega i}$ may be defined as functions of own state variables and time, as well as on the coordinates of its neighbors. It is furthermore assumed that the interconnections with the latter form a connected, undirected, and static graph whose connectivity is defined by the Laplacian matrix $L:=\left[\ell_{i j}\right] \in$ $\mathbb{R}^{N \times N}$, where

$$
\ell_{i j}=\left\{\begin{array}{cc}
\sum_{j \in \mathcal{N}_{i}} a_{i j} & i=j \\
-a_{i j} & i \neq j
\end{array}\right.
$$

and $a_{i j} \geq 0 ; a_{i j}>0$ if the $i$ th and $j$ th vehicles communicate with each other and $a_{i j}=0$ otherwise. Since the graph is undirected, $a_{i j}=a_{j i}$, so $L$ is symmetric and, by construction, $L \mathbf{1}_{N}=0$, where $\mathbf{1}_{N} \in \mathbb{R}^{N}$. Also, $L$ has a single zero-eigenvalue and the rest of the spectrum of $L$ is positive. Thus, $\operatorname{rank}(L)=N-1$.

For such a network of $N$ vehicles we address the following consensus-formation control problem. Let $\left(x_{c}, y_{c}\right) \in \mathbb{R}^{2}$ denote the Cartesian coordinates of an a prioiri unknown center in the plane and, for each robot, let $\left(\delta_{x_{i}}, \delta_{y_{i}}\right)$ be given. Then, we define partial consensus as the goal of rendering the set

$$
\begin{aligned}
\mathcal{S}_{p c}:=\bigcup_{i \leq N}\left\{v_{i}=0, \omega_{i}=0, \theta_{i}=\theta_{d i},\right. \\
{\left.\left[x_{i}-\delta_{x_{i}}\right]=x_{c},\left[y_{i}-\delta_{y_{i}}\right]=y_{c}\right\} }
\end{aligned}
$$

uniformly globally asymptotically stable.

Partial consensus, as defined above, comprises the property that the robots stabilize in formation around an unknown Cartesian point. That is the property that

$$
\begin{gathered}
\lim _{t \rightarrow \infty} x_{i}(t):=x_{c}+\delta_{x i}, \quad \lim _{t \rightarrow \infty} y_{i}(t):=y_{c}+\delta_{y i}, \\
\lim _{t \rightarrow \infty} \theta_{i}(t):=\theta_{d i}, \quad \forall i \leq N
\end{gathered}
$$

which means, only, non-uniform convergence.

We solve the partial consensus problem as one of stabilization. To that end, we first introduce a dynamical model of the interconnected vehicles that is more suitable for control. Consider the error coordinates

$$
z_{i}:=\left[\begin{array}{c}
x_{i}-\delta_{x_{i}} \\
y_{i}-\delta_{y_{i}}
\end{array}\right], \quad z:=\left[z_{1}^{\top} \cdots z_{N}^{\top}\right]^{\top}
$$

and, to compact the notation, let us introduce as well the vectors $\theta=\left[\theta_{1} \cdots \theta_{N}\right]^{\top} \in \mathbb{R}^{N} ; v=\left[v_{1} \cdots v_{N}\right]^{\top} \in \mathbb{R}^{N} ; \omega=$ $\left[\omega_{1} \cdots \omega_{N}\right]^{\top} \in \mathbb{R}^{N}, \Phi(\theta)=\operatorname{blockdiag}\left[\phi\left(\theta_{i}\right)\right] \in \mathbb{R}^{2 N \times N}$, with $\phi\left(\theta_{i}\right)=\left[\cos \left(\theta_{i}\right) \sin \left(\theta_{i}\right)\right]^{\top}$, and the control inputs $u_{v}=\left[u_{v 1} \cdots u_{v N}\right]^{\top} \in \mathbb{R}^{N}$ and $u_{\omega}=\left[u_{\omega 1} \cdots u_{\omega N}\right]^{\top} \in \mathbb{R}^{N}$. In terms of these variables the dynamics of the overall openloop system is

$$
\begin{aligned}
\dot{z} & =\Phi(\theta) v \\
\dot{v} & =u_{v} \\
\dot{\theta} & =\omega \\
\dot{\omega} & =u_{\omega} .
\end{aligned}
$$

The first two equations determine the translational dynamics while the second pair defines the angular motion, so partial consensus is reached if the equilibrium point

$$
\{(z, \theta, v, \omega)\}=\left\{\left(\mathbf{1}_{N} \otimes z_{c}, \theta_{d}, 0,0\right)\right\},
$$

where $\mathbf{1}_{N}=\left[\begin{array}{lll}1 & \cdots & 1\end{array}\right]^{\top}$ and $z_{c}:=\left[\begin{array}{ll}x_{c} & y_{c}\end{array}\right]^{\top}$ corresponds to the (non-a priori defined) center of the formation pattern, is uniformly globally asymptotically stable.

Now, the condition $z=\mathbf{1}_{N} \otimes z_{c}$ may be characterized in terms of the relative position errors between each vehicle and its neighbors. For this purpose, we introduce the errors

$$
\begin{aligned}
e_{i} & =\phi\left(\theta_{i}\right)^{\top} \sum_{j \in \mathcal{N}_{i}} a_{i j}\left(z_{i}-z_{j}\right), \\
s_{i} & =\phi\left(\theta_{i}\right)^{\perp \top} \sum_{j \in \mathcal{N}_{i}} a_{i j}\left(z_{i}-z_{j}\right)
\end{aligned}
$$

$\phi\left(\theta_{i}\right)^{\perp}=\left[\sin \left(\theta_{i}\right)-\cos \left(\theta_{i}\right)\right]^{\top}$ or, in compact form,

$$
e=\Phi(\theta)^{\top} \mathcal{L} z, \quad s=\Phi(\theta)^{\perp \top} \mathcal{L} z,
$$

where $\mathcal{L}:=L \otimes I_{2}, \Phi(\theta)^{\perp}=\operatorname{diag}\left[\phi\left(\theta_{i}\right)^{\perp}\right] \in \mathbb{R}^{2 N \times N}$, $e:=\left[e_{1}^{\top} \cdots e_{N}^{\top}\right]^{\top}$, and $s:=\left[s_{1}^{\top} \cdots s_{N}^{\top}\right]^{\top}$. Note that since the matrix $\left[\Phi(\theta) \Phi(\theta)^{\perp}\right]$ is non singular and the communication graph is connected, we have

$$
\mathcal{L} z=0 \Leftrightarrow(e, s)=(0,0)
$$

or, equivalently, $(e, s)=(0,0)$ if and only if $z=\mathbf{1}_{N} \otimes z_{c}$. Therefore, the partial consensus objective is reached if and only if the equilibrium $\{(v, e, s, \omega, \theta)\}=\left\{\left(0,0,0,0, \theta_{d}\right)\right\}$ is rendered uniformly globally asymptotically stable. We establish this next, via a decentralized controller.

\section{MAIN RESUlTS}

In [22], the following $\delta$-persistently-exciting controller was proposed to solve the partial-consensus problem in the absence of delays:

$$
\begin{aligned}
& u_{v}=-K_{d t} v-K_{p t} e, \\
& u_{\omega}=-K_{d \theta} \omega-K_{p \theta} \tilde{\theta}-p(t) \kappa(s, e),
\end{aligned}
$$

where $K_{d t}, K_{p t}, K_{d \theta}$, and $K_{p \theta}$ are diagonal positive definite matrices, $\tilde{\theta}:=\theta-\theta_{d}$, for each $i \leq N$, e :=[e $\left.e_{1}, \cdots e_{N}\right]^{\top}$ and $s:=\left[s_{1}, \cdots s_{N}\right]^{\top}$ are the measured errors,

$$
\kappa(s, e)=\frac{1}{2}\left[s_{1}^{2}+e_{1}^{2}, \cdots, s_{N}^{2}+e_{N}^{2}\right]^{\top},
$$


and $p$ is constructed as a function with persistently exciting first derivative that is, such that there exist $T_{p}$ and $\mu_{p}>0$ such that

$$
\int_{t}^{t+T_{p}}|\dot{p}(\tau)| d \tau \geq \mu_{p} \quad \forall t \geq 0 .
$$

Persistency of excitation is a well-known property mostly used in adaptive control and system identification; it is used to ensure that the parameter estimation errors converge to zero [24]. Here, the control mechanism that overcomes the structural obstruction imposed by the nonholonomic nature of the unicycle is a property called $\delta$-persistency of excitation [25] and it is used in the control action to overcome the difficulties due to the nonholonomy $-c f$. [1]. Thus, the term $p(t) \kappa(s, e)$ is an essential component of the control law (13), which works in a similar fashion as persistency of excitation does in adaptive control. More precisely, the control action relies on the term $p(t) \kappa(s, e)$ being $\delta$-persistently-exciting, that is, on the existence, for any $\delta>0$, of $T_{\delta}$ and $\mu_{\delta}>0$ such that

$$
\left|\left[\begin{array}{l}
e \\
s
\end{array}\right]\right| \geq \delta \Longrightarrow \int_{t}^{t+T_{\delta}}|\kappa(e, s) p(\tau)| d \tau \geq \mu_{\delta} \quad \forall t \geq 0 .
$$

In view of (14), the property (16) may be interpreted as $p(t) \kappa(s, e)$ being persistently exciting "as long as" the error $(e, s)$ is away from zero, that is, while consensus has not been reached. It is important to notice, however, that $(e, s)$ in the integrand above are elements of $\mathbb{R}^{N} \times \mathbb{R}^{N}$; the integral above is not evaluated along the system's trajectories.

In this paper, we employ the following certaintyequivalence controller that stabilizes the system in the presence of time-varying bounded measurement delay (boundedness of the variation of the delay is not needed):

$$
\begin{aligned}
& u_{v}=-K_{d t} v-K_{p t} e_{d}, \\
& u_{\omega}=-K_{d \theta} \omega-K_{p \theta} \tilde{\theta}-p(t) \kappa\left(s_{d}, e_{d}\right),
\end{aligned}
$$

where $e_{d}:=\left[e_{d 1}, \cdots e_{d N}\right]^{\top}$ and $s_{d}:=\left[s_{d 1}, \cdots s_{d N}\right]^{\top}$ denote the measured errors affected by bounded delays $t \mapsto$ $T_{i j}(t)$, that is,

$$
\begin{aligned}
& e_{d i}=\phi\left(\theta_{i}\right)^{\top} \sum_{j \in \mathcal{N}_{i}} a_{i j}\left(z_{i}(t)-z_{j}\left(t-T_{i j}(t)\right)\right), \\
& s_{d i}=\phi\left(\theta_{i}\right)^{\perp \top} \sum_{j \in \mathcal{N}_{i}} a_{i j}\left(z_{i}(t)-z_{j}\left(t-T_{i j}(t)\right)\right) .
\end{aligned}
$$

Proposition 1 (Main result) Consider the system (7) in closed loop with the controller (17)-(18) with $K_{d t}, K_{p t}$, $K_{d \theta}$, and $K_{p \theta}$ diagonal positive definite. Assume that there exists $b_{p}>0$ such that

$$
\max \left\{|p|_{\infty},|\dot{p}|_{\infty},|\ddot{p}|_{\infty},\left|p^{(3)}\right|_{\infty}\right\} \leq b_{p}
$$

where, $|\varphi|_{\infty}:=\sup _{t \geq 0}|\varphi(t)|$ and, moreover, $\dot{p}(t)$ is persistently exciting with excitation parameters $\left(T_{p}, \mu_{p}\right)$. If, in addition, there exists $T^{*}>0$ such that $T_{i j}(t) \in\left[0, T^{*}\right]$ for all $i, j \leq N, t \geq 0$ and the matrices $K_{d t}$ and $K_{p t}$ satisfy

$$
1-\left(1+N^{2} \bar{a}^{2}\right) T^{*} \lambda_{\max }\left(K_{p t} K_{d t}^{-1}\right) \geq 0,
$$

where $\bar{a}:=\max \left\{a_{i j}\right\}$, then $\{(e, s, v, \tilde{\theta}, \omega)\}=$ $\{(0,0,0,0,0)\}$ is uniformly globally asymptotically stable.

Sketch of proof. Firstly, we write the error-dynamics equations. For $e$ and $s$, these are obtained by differentiating on both sides of the the two equations in (10) and using (7a) and

$$
\dot{\Phi}(\theta)=-\Phi(\theta)^{\perp} \bar{\omega}, \quad \dot{\Phi}(\theta)^{\perp}=\Phi(\theta) \bar{\omega},
$$

where $\bar{\omega}:=\operatorname{diag}\left[\omega_{i}\right] \in \mathbb{R}^{N \times N}$, to obtain

$$
\begin{aligned}
\dot{e} & =-\bar{\omega} s+\Phi(\theta)^{\top} \mathcal{L} \Phi(\theta) v \\
\dot{s} & =\bar{\omega} e+\Phi(\theta)^{\perp \top} \mathcal{L} \Phi(\theta) v .
\end{aligned}
$$

Then, by direct substitution of (17) and (18) in (7b) and (7d) respectively, we obtain

$$
\begin{aligned}
\dot{v} & =-K_{d t} v-K_{p t} e_{d} \\
\dot{\tilde{\theta}} & =\omega \\
\dot{\omega} & =-K_{d \theta} \omega-K_{p \theta} \tilde{\theta}-p(t) \kappa\left(s_{d}, e_{d}\right) .
\end{aligned}
$$

For further development, it is useful to stress that, after (19) and (20), $e_{d}$ and $s_{d}$ may be expressed in function of $e$ and $s$ as

$$
\begin{aligned}
& e_{d}=e+\Phi(\theta)^{\top} \mathcal{A}\left(\dot{z}_{t}\right), \\
& s_{d}=s+\Phi(\theta)^{\perp \top} \mathcal{A}\left(\dot{z}_{t}\right)
\end{aligned}
$$

where $\dot{z}_{t}$ is short notation for $\dot{z}_{t}(\Delta):=\dot{z}(t+\Delta)$, with $\Delta \in$ $\left[-T^{*}, 0\right]$ and

$$
\mathcal{A}\left(\dot{z}_{t}\right):=\left[\begin{array}{c}
\mathcal{A}_{1}\left(\dot{z}_{t}\right) \\
\vdots \\
\mathcal{A}_{N}\left(\dot{z}_{t}\right)
\end{array}\right], \quad \mathcal{A}_{i}\left(\dot{z}_{t}\right)=\sum_{j \in \mathcal{N}_{i}} a_{i j} \int_{t-T_{j i}(t)}^{t} \dot{z}_{j}(\tau) d \tau .
$$

Furthermore, $\kappa\left(e_{d}, s_{d}\right)$ in (27b) may be expressed as

$$
\kappa\left(e_{d}, s_{d}\right)=\kappa(e, s)+\kappa_{d}\left(e, s, \theta, \dot{z}_{t}\right)
$$

where

$$
\kappa_{d}=\frac{1}{2}\left[\begin{array}{c}
\mathcal{A}_{1}^{\top} \mathcal{A}_{1}+2 e_{1} \phi_{1}^{\top} \mathcal{A}_{1}+2 s_{1} \phi_{1}^{\perp \top} \mathcal{A}_{1} \\
\vdots \\
\mathcal{A}_{N}^{\top} \mathcal{A}_{N}+2 e_{N} \phi_{N}^{\top} \mathcal{A}_{N}+2 s_{N} \phi_{N}^{\perp \top} \mathcal{A}_{N}
\end{array}\right] .
$$

That is, in the absence of delays, i.e., with $\mathcal{A}_{i}^{\top}\left(\dot{z}_{t}\right)=0$, we recover the error-dynamics equations from [26].

Next, for the purpose of analysis, let

$$
e_{\theta}:=\tilde{\theta}+q(t) \kappa(s, e), \quad e_{\omega}:=\omega+\dot{q}(t) \kappa(s, e)
$$

where $q: \mathbb{R}_{\geq 0} \rightarrow \mathbb{R}^{N \times N}$ is a differentiable function defined dynamically as

$$
\ddot{q}+K_{d \theta} \dot{q}+K_{p \theta} q=p(t) I_{N}
$$

and, in turn, $K_{d \theta}$ and $K_{p \theta}$ are diagonal positive definite matrices. We stress that, after (21) there exists $b_{q}>0$ such that

$$
\max \left\{|q|_{\infty},|\dot{q}|_{\infty},|\ddot{q}|_{\infty},\left|q^{(3)}\right|_{\infty}\right\} \leq b_{q}
$$


and, since $\dot{q}$ is solution to

$$
q^{(3)}+K_{d \theta} \ddot{q}+K_{p \theta} \dot{q}=\dot{p}(t) I_{N}
$$

and $\dot{p}$ is persistently exciting by assumption, so is $\dot{q}-[24]$.

In the new equivalent coordinates (32), the closed-loop dynamics equations become

$$
\begin{gathered}
\dot{X}_{t}=\left[\begin{array}{ccc}
-K_{d t} & -K_{p t} & 0 \\
0 & 0 & \dot{q} \bar{\kappa}-\bar{e}_{\omega} \\
0 & -\dot{q} \bar{\kappa}+\bar{e}_{\omega} & 0
\end{array}\right] X_{t} \\
+\left[\begin{array}{c}
0 \\
\Phi^{\top} \mathcal{L} \\
\Phi^{\perp \top} \mathcal{L}
\end{array}\right] \Phi v-\left[\begin{array}{c}
K_{p t} \Phi^{\top} \\
0 \\
0
\end{array}\right] \mathcal{A}\left(\dot{z}_{t}\right) \\
\dot{X}_{r}=\left[\begin{array}{cc}
0 & I_{N} \\
-K_{p \theta} & -K_{d \theta}
\end{array}\right] X_{r} \\
+\left[\begin{array}{l}
q \\
\dot{q}
\end{array}\right]\left[\begin{array}{l}
\left.\bar{e} \Phi^{\top}+\bar{s} \Phi^{\perp \top}\right] \mathcal{L} \Phi v-\left[\begin{array}{l}
0 \\
p
\end{array}\right] \kappa_{d}
\end{array}\right.
\end{gathered}
$$

where $\bar{\kappa}:=\operatorname{diag}\left[s_{i}^{2}+e_{i}^{2}\right], \bar{e}_{\omega}:=\operatorname{diag}\left[e_{\omega_{i}}\right], X_{t}:=\left[v^{\top} e^{\top} s^{\top}\right]^{\top}$ and $X_{r}:=\left[e_{\theta}^{\top} e_{\omega}^{\top}\right]^{\top}$.

The rest of the proof consists in establishing uniform global asymptotic stability of the origin for (35). This may be accomplished by arguing as in the proof of the generalized Matrosov's theorem [25]. First, we need to establish uniform global stability (namely, that the solutions are uniformly globally bounded and the origin is uniformly Lyapunov stable). This, in turn, may be accomplished in two separate steps, for (35a) and (35b). For the former, we use a Lyapunov-Krasovskii functional that is reminiscent of the strict Lyapunov function proposed in [26] for the system (24)-(27) subject to $\left(e_{d}, s_{d}\right)=(e, s)$, that is, without delays. Let

$$
V\left(v, z, \dot{z}_{t}\right)=v^{\top} K_{p t}^{-1} v+z^{\top} \mathcal{L} z+\int_{-T^{*}}^{0} \int_{t+\theta}^{t}|\dot{z}(\tau)|^{2} d \tau d \theta .
$$

After [22, Lemma 1], we have

$$
\lambda_{2}(L) z^{\top} \mathcal{L} z \leq|e|^{2}+|s|^{2} \leq \lambda_{N}(L) z^{\top} \mathcal{L} z,
$$

so, in view of the inequality

$$
\int_{-T^{*}}^{0} \int_{t+\theta}^{t}|\dot{z}(\tau)|^{2} d \tau d \theta \leq T^{*} \int_{t-T^{*}}^{t}|\dot{z}(\tau)|^{2} d \tau
$$

it follows, according to [27], that the functional $V$ is positive definite and radially unbounded with respect to $X_{t}$. Furthermore, the time-derivative of $V$ along the trajectories of (35a) yields

$$
\begin{aligned}
\dot{V} & \leq-v^{\top} K_{p t}^{-1} K_{d t} v-Y\left(\dot{z}_{t}\right) \\
Y\left(\dot{z}_{t}\right) & :=\frac{1}{2 \bar{a}^{2} N T^{*}} \sum_{j=1}^{N} \sum_{i=1}^{N} a_{i j}^{2}\left|\int_{t-T_{i j}}^{t} \dot{z}_{i}(\tau) d \tau\right|^{2}
\end{aligned}
$$

which is negative semidefinite. This establishes uniform global stability for (35a) and, integrating on both sides of $\dot{V} \leq-v^{\top} K_{p t}^{-1} K_{d t} v$, we also conclude that $v$ and $Y\left(z_{t}\right)$, consequently also $\kappa_{d}$, tend to zero asymptotically. Note, moreover, that (35a) is input-to-state stable with respect to the converging input $\left(v, \kappa_{d}\right)$, so uniform global stability follows.

To establish uniform convergence, according to [25], we need to find a series of functions $V_{i}$ and $\psi_{i}$ such that $\dot{V}_{i} \leq$ $\psi_{i}\left(t, X_{t}, X_{r}\right)$ and having the properties that each $\psi_{i} \leq 0$ on the set where $\psi_{j}=0$ for all $j \leq i$ and the only point at which $\psi_{i}\left(t, X_{t}, X_{r}\right) \equiv 0, \forall i$, is the origin. This property is verified as follows.

Let the first function $V_{1}$, correspond to $V\left(v, z, \dot{z}_{t}\right)$ defined in (36). Then, $\psi_{1}:=-v^{\top} K_{p t}^{-1} K_{d t} v-Y\left(\dot{z}_{t}\right)$. Now, let $V_{2}(e, v):=e^{\top} v$; its total derivative,

$$
\dot{V}_{2}=v^{\top} \Phi^{\top} \mathcal{L} \Phi v+s^{\top} \dot{q} \bar{\kappa} v-e^{\top} K_{d t} v-e^{\top} K_{p t} e-v^{\top} \bar{e}_{\omega} s,
$$

satisfies $\dot{V}_{2}=-e^{\top} K_{p t} e=: \psi_{2} \leq 0$ on the set $\left\{\psi_{1}=0\right\}$.

Then, we introduce

$$
V_{3}\left(e_{\theta}, e_{\omega}\right)=c_{2}\left[e_{\omega}^{\top} e_{\omega}+e_{\theta}^{\top} K_{p \theta} e_{\theta}\right]+e_{\theta}^{\top} e_{\omega} .
$$

Note that $Y\left(\dot{z}_{t}\right)=0$ implies that $\kappa_{d}=0$ hence, the total derivative of $V_{3}$, restricted to the set $\left\{Y\left(\dot{z}_{t}\right)=0\right\}$, satisfies

$$
\begin{aligned}
\dot{V}_{3} \leq & -\left[\frac{c_{2}}{4} e_{\omega}^{\top} K_{d \theta} e_{\omega}+\frac{1}{4} e_{\theta}^{\top} K_{p \theta} e_{\theta}\right] \\
& +\frac{c_{2}}{2}\left|\bar{e} \Phi^{\top} \mathcal{L} \Phi v\right|^{2}+\frac{c_{2}}{2}\left|\bar{s} \Phi^{\perp \top} \mathcal{L} \Phi v\right|^{2},
\end{aligned}
$$

That is,

$$
\dot{V}_{3} \leq-\frac{1}{4}\left[c_{2} e_{\omega}^{\top} K_{d \theta} e_{\omega}+e_{\theta}^{\top} K_{p \theta} e_{\theta}\right] \leq 0
$$

on the set $\left\{\psi_{1}=0\right\} \cap\left\{\psi_{2}=0\right\}$, so we define $\psi_{3}:=$ $-\frac{1}{4}\left[c_{2} e_{\omega}^{\top} K_{d \theta} e_{\omega}+e_{\theta}^{\top} K_{p \theta} e_{\theta}\right]$ and we carry on.

Let $V_{41}(t, e, s):=e^{\top} \dot{q}(t) s$; its total derivative yields

$$
\begin{gathered}
\dot{V}_{41}=-e^{\top} \ddot{q} s-v^{\top} \Phi^{\top} \mathcal{L} \Phi \dot{q} s-s^{\top} \bar{\kappa} \dot{q}^{2} s+e^{\top} \dot{q}^{2} \bar{\kappa} e \\
-e^{\top} \dot{q} \Phi^{\perp \top} \mathcal{L} \Phi v+s^{\top} \bar{e}_{\omega} \dot{q} s-e^{\top} \dot{q} \bar{e}_{\omega} e
\end{gathered}
$$

and on the set $\left\{\psi_{1}=0\right\} \cap\left\{\psi_{2}=0\right\} \cap\left\{\psi_{3}=0\right\}$, which is equivalent to $\left\{v=0, Y\left(\dot{z}_{t}\right)=0, e=0, e_{\theta}=0, e_{\omega}=0\right\}$ satisfies $\dot{V}_{41}=-s^{\top} \bar{\kappa} \dot{q}^{2} s \leq 0$.

Next, we introduce the function

$$
\begin{aligned}
V_{42}(t, s, e) & :=\kappa(s, e)^{\top} \Upsilon(t) \kappa(s, e) \\
\Upsilon(t) & :=1+2 b_{q}^{2} T I_{N}-\frac{2}{T} \int_{t}^{t+T} \int_{t}^{m} \dot{q}(\tau)^{2} d \tau d m,
\end{aligned}
$$

which has the following properties. First, $|\Upsilon(t)| \leq 1+2 b_{q}^{2} T^{*}$. Second,

$$
\dot{\Upsilon} \leq-\frac{2}{T} \int_{t}^{t+T} \dot{q}(\tau)^{2} d \tau \leq-\frac{2 \mu}{T} I_{N}
$$

The last inequality follows from the property that $\dot{q}$ is persistently exciting.

On the other hand,

$$
\begin{aligned}
\dot{V}_{42}=- & \kappa^{\top} \frac{2}{T} \int_{t}^{t+T} \dot{q}(s)^{2} d s \kappa+\kappa^{\top} \dot{q}(t)^{2} \kappa \\
& +2 \kappa^{\top} \Upsilon\left[\bar{e} \Phi^{\top} \mathcal{L} \Phi v+\bar{s} \Phi^{\perp \top} \mathcal{L} \Phi v\right]
\end{aligned}
$$

and, by virtue of the fact that

$$
\kappa^{\top} \dot{q}(t)^{2} \kappa \leq s^{\top} \dot{q}(t)^{2} \bar{\kappa} s+b_{q}^{2} \lambda_{N}(L) V^{2}\left|K_{p t}^{-1}\right| e^{\top} K_{p t} e,
$$


we conclude that, defining $V_{4}:=V_{41}+V_{42}$,

$$
\dot{V}_{4} \leq-\frac{2 \mu}{T}|\kappa|^{2}
$$

on the set $\left\{\psi_{1}=0\right\} \cap\left\{\psi_{2}=0\right\} \cap\left\{\psi_{3}=0\right\}$. Thus, we see that

$$
\bigcap_{i \leq 4}\left\{\psi_{i} \leq 0\right\}=\left\{\left(e, s, v, e_{\omega}, e_{\theta}\right)=(0,0,0,0,0)\right\}
$$

so the result follows.

\section{A NUMERICAL EXAMPLE}

In this section we illustrate our main result via numerical simulations. The example that we present concerns four identical robots that are required to achieve consensus in their Cartesian coordinates and form a rhomboid shape. This is achieved by choosing the offsets $\left(\delta_{x i}, \delta_{y i}\right)$ as in showed in Table I below. The table also contains the initial conditions of the robots. The desired values or the orientation angles (in [rad] ) were set to $\theta_{d}=\left[\begin{array}{llll}-1 & 1 & 0 & -0.5\end{array}\right]^{\top}$.

TABLE I

INITIAL CONDITIONS, RELATIVE DESIRED POSITIONS AND DESIRED ORIENTATIONS.

\begin{tabular}{c|c|c|c|c|c|c|}
\cline { 2 - 7 } & $x_{i}(0)$ & $y_{i}(0)$ & $\theta_{i}(0)$ & $\delta_{x_{i}}$ & $\delta_{y_{i}}$ & $\theta_{d i}$ \\
\hline 1 & 5 & -4 & $-(1 / 3) \pi$ & -2 & 0 & 0 \\
\hline 2 & -4 & 5 & $(2 / 3) \pi$ & 0 & 2 & $-\pi / 2$ \\
\hline 3 & -2 & -5 & $(4 / 3) \pi$ & 2 & 0 & $-\pi$ \\
\hline 4 & 7 & 3 & 0 & 0 & -2 & $\pi / 2$ \\
\hline
\end{tabular}

The robots are interconnected in a closed undirected cycle as illustrated in Fig. 1, where the Laplacian matrix is also given. The control gains have been set to: $K_{d t}=60 \mathbf{I}_{4}$;

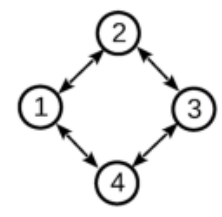

$$
L=\left[\begin{array}{rrrr}
2 & -1 & 0 & -1 \\
-1 & 2 & -1 & 0 \\
0 & -1 & 2 & -1 \\
-1 & 0 & -1 & 2
\end{array}\right]
$$

Fig. 1. Interconnection Graph and Laplacian matrix

$K_{p t}=30 \mathbf{I}_{4} ; K_{d \theta}=30 \mathbf{I}_{4}$; and $K_{p \theta}=10 \mathbf{I}_{4}$. The persistently exciting function $p(t)$ is set to a rich signal of four frequencies, (as many as robots in the network):

$p(t)=-\frac{5}{2}+2 \pi \sin (0.5 * t)+\pi \sin (2 t)+3 \pi \sin (3 t)+\pi \sin (t)$.

For simplicity, the time-varying delay is the same for all the interconnections and is depicted in Fig. 2, its bound has been taken as $T *=0.37$. These delays follow a normal Gaussian distribution with a mean of 0.3 and a variance of 0.0003 .

In Fig. 3 are depicted the paths in the plane described by the robots. The initial positions are marked by '*' and the final orientations are represented by arrows. In Figs. 4 and
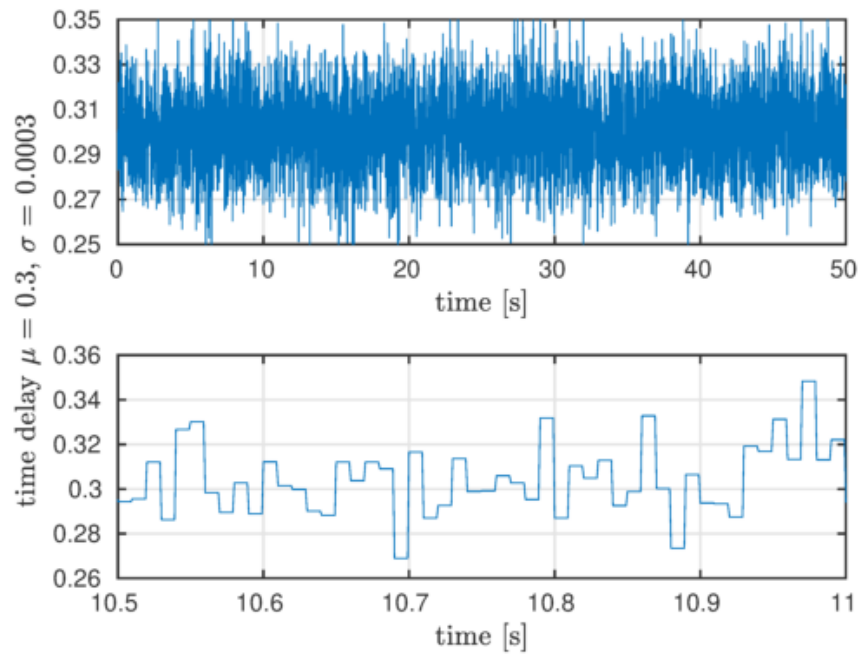

Fig. 2. Time delay in the network interconnection.

5 are depicted the robots' relative positions, $\bar{z}_{i}$, and their orientations. It can be observed that the robots reach the desired formation with an a priori unknown center at $z_{c} \approx$ $(1.68,-0.6)$ and, as required, all robots point at it.



Fig. 3. Trajectories and formation of the network of mobile robots.

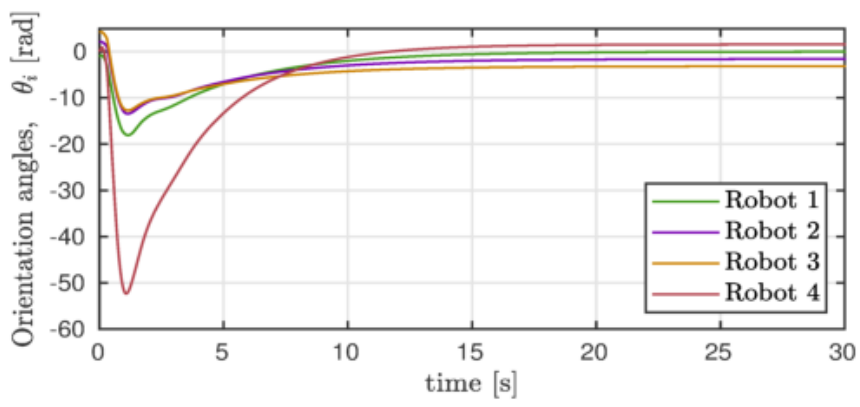

Fig. 4. Orientation of each mobile robot in the network. 

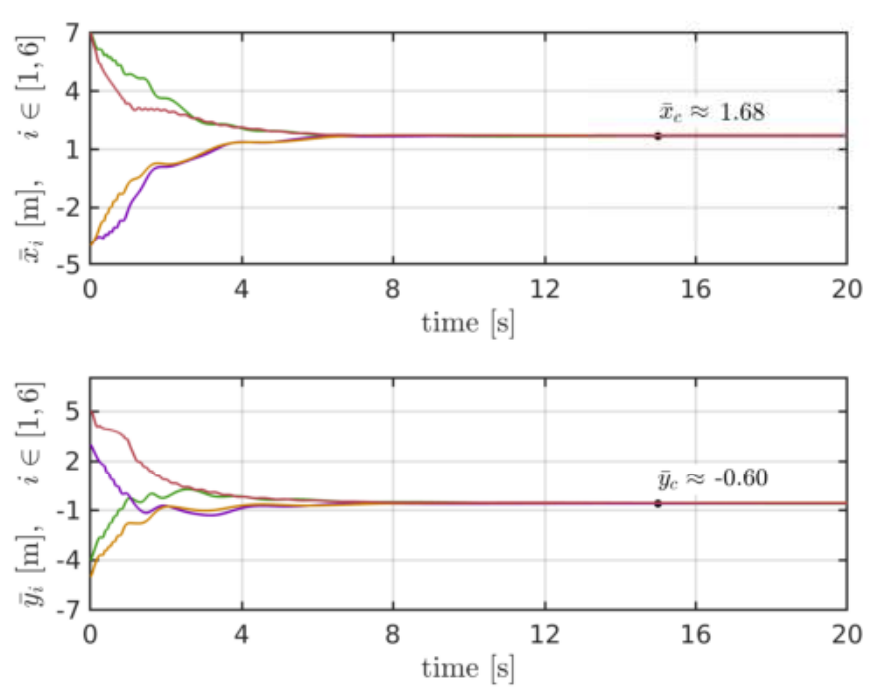

Fig. 5. Evolution of the relative positions $z_{i}$.

\section{Conclusions}

A fairly simple decentralized controller for partial consensus of nonholonomic mobile robots in the presence of non smooth time-varying communication delays was presented. The controller is of the proportional-plus-derivative type and relies on $\delta$-persistency of excitation to overcome the effects of nonholonomy.

This controller applies in the common scenario in which a group of robots, equiped with global positioning sensors, establish a bidirectional communication over a wireless network. The communication, however, may be affected by diffent time-varying delays. Our main result, however, does not account for possible obstacles and it does not apply if some states are not measurable. Current research is being carried in this direction.

\section{REFERENCES}

[1] A. Loria, E. Panteley, and A. Teel, "A new persistency-of-excitation condition for UGAS of NLTV systems: Application to stabilization of nonholonomic systems," in Proc. of the 5th. European Control Conf., (Karlsrühe, Germany), pp. 1363-1368, 1999.

[2] W. Ren and R. W. Beard, Distributed consensus in multivehicle cooperative control. Springer verlag, 2005.

[3] W. Ren and E. Atkins, "Distributed multi-vehicle coordinated control via local information exchange," International Journal of Robust and Nonlinear Control, vol. 17, no. 10-11, pp. 1002-1033, 2007.

[4] E. Nuño, R. Ortega, L. Basañez, and D. Hill, "Synchronization of networks of nonidentical Euler-Lagrange systems with uncertain parameters and communication delays," IEEE Trans. on Automatic Control, vol. 56, no. 4, pp. 935-941, 2011.

[5] W. Ren and R. W. Beard, "Consensus seeking in multiagent systems under dynamically changing interaction topologies," IEEE Trans. on Automatic Control, vol. 50, no. 5, pp. 655-661, 2005.

[6] S. Martin and A. Girard, "Continuous-time consensus under persistent connectivity and slow divergence of reciprocal interaction weights," SIAM Journal on Control and Optimization, vol. 51, no. 3, pp. 25682584, 2013.

[7] A. Abdessameud, I. G. Polushin, and A. Tayebi, "Synchronization of Lagrangian systems with irregular communication delays," IEEE Trans. on Automatic Control, vol. 59, no. 1, pp. 187-193, 2014.
[8] E. Nuño, I. Sarras, and L. Basañez, "Consensus in networks of nonidentical Euler-Lagrange systems using $\mathrm{P}+\mathrm{d}$ controllers," IEEE Trans. on Robotics, vol. 26, no. 6, pp. 1503-1508, 2013.

[9] Z. Li, W. Ren, X. Liu, and M. Fu, "Consensus of multi-agent systems with general linear and lipschitz nonlinear dynamics using distributed adaptive protocols," IEEE Trans. on Automatic Control, vol. 58, no. 7, pp. 1786-1791, 2013.

[10] Q. Hui, W. M. Haddad, and S. P. Bhat, "Finite-time semistability and consensus for nonlinear dynamical networks," IEEE Trans. on Automatic Control, vol. 53, no. 8, pp. 1887-1900, 2008.

[11] E. Panteley and A. Loria, "Synchronization and dynamic consensus of heterogeneous networked systems," IEEE Trans. on Automatic Control, vol. 62, no. 8, pp. 3758-3773, 2017.

[12] P. Wieland and F. Allgöwer, "An internal model principle for consensus in heterogeneous linear multi-agent systems," in Estimation and Control of Networked Systems, pp. 7-12, 2009.

[13] Z. Lin, B. Francis, and M. Maggiore, "Necessary and sufficient graphical conditions for formation control of unicycles," IEEE Trans. on Automatic Control, vol. 50, no. 1, pp. 121-127, 2005.

[14] D. V. Dimarogonas and K. J. Kyriakopoulos, "On the rendezvous problem for multiple nonholonomic agents," IEEE Trans. on Automatic Control, vol. 52, no. 5, pp. 916-922, 2007.

[15] C. Yang, W. Xie, C. Lei, and B. Ma, "Smooth time-varying formation control of multiple nonholonomic agents," in Proc. of the 2015 Chinese Intelligent Systems Conference, pp. 283-291, Springer, 2016.

[16] Z. Peng, G. Wen, A. Rahmani, and Y. Yu, "Distributed consensusbased formation control for multiple nonholonomic mobile robots with a specified reference trajectory," International Journal of Systems Science, vol. 46, no. 8, pp. 1447-1457, 2015.

[17] E. Nuño, T. Hernández, M. Maghenem, A. Loría, and E. Panteley, "Leaderless consensus-based formation control of multiple nonholonomic mobile robots with interconnecting delays," in Proc. IEEE American Control Conference, (Philadelphia, PA, USA), pp. 46594664, 2019.

[18] W. Dong and J. A. Farrell, "Consensus of multiple nonholonomic systems," in Proc. of the 47th IEEE Conf. on Decision and Control, pp. 2270-2275, IEEE, 2008.

[19] A. Ajorlou, M. Asadi, A. G. Aghdam, and S. Blouin, "Distributed consensus control of unicycle agents in the presence of external disturbances," Systems \& Control Letters, vol. 82, pp. 86-90, 2015.

[20] A. Loria, E. Panteley, and K. Melhem, "UGAS of skew-symmetric time-varying systems: application to stabilization of chained form systems," European Journal of Control, vol. 8, no. 1, pp. 33-43, 2002.

[21] Y. Wang, Z. Miao, H. Zhong, and Q. Pan, "Simultaneous stabilization and tracking of nonholonomic mobile robots: A Lyapunov-based approach," IEEE Trans. on Control Systems Technology, vol. 23, pp. 1440-1450, July 2015.

[22] M. Maghenem, A. Bautista-Castillo, E. Nuño, A. Loria, and E. Panteley, "Consensus of multi-agent systems with nonholonomic restrictions via Lyapunov's direct method," IEEE Control Systems Letters, vol. 3, no. 2, pp. 344-349, 2019. DOI: 10.1109/LCSYS.2018.2879043.

[23] K. D. Do, Z.-P. Jiang, and J. Pan, "A global output-feedback controller for simultaneous tracking and stabilization of unicycle-type mobile robots," IEEE Trans. on Robotics Automat., vol. 20, no. 3, pp. 589594, 2004.

[24] P. Ioannou and J. Sun, Robust adaptive control. New Jersey, USA: Prentice Hall, 1996.

[25] A. Loria, E. Panteley, D. Popović, and A. Teel, "A nested Matrosov theorem and persistency of excitation for uniform convergence in stable non-autonomous systems," IEEE Trans. on Automatic Control, vol. 50, no. 2, pp. 183-198, 2005.

[26] M. Maghenem, A. Bautista-Castillo, E. Nuño, A. Loria and E. Panteley, "Consensus-based formation control of nonholonomic robots using a strict Lyapunov function," IFAC-PapersOnLine, vol. 50, no. 1, pp. 2439-2444, 2017.

[27] E. Fridman, "Tutorial on Lyapunov-based methods for time-delay systems," European Journal of Control, vol. 20, no. 6, pp. 271-283, 2014. 